Accepted Dec. 2102 _Solid State Sciences

\title{
First principles study of the electronic and magnetic structures and
} bonding properties of $\mathrm{UCoC}_{2}$ ternary, characteristic of $\mathrm{C}-\mathrm{C}$ units.

\author{
Samir F. Matar ${ }^{\mathrm{a}, \mathrm{b}, *}$ \\ ${ }^{a}$ CNRS, ICMCB, UPR 9048, F-33600 Pessac, France. \\ ${ }^{\mathrm{b}}$ Univ. Bordeaux, ICMCB, UPR 9048, F-33600 Pessac, France. \\ *e_mail: matar@icmcb-bordeaux.cnrs.fr
}

\begin{abstract}
The electronic structure of $\mathrm{UCoC}_{2}$, a di-carbide with the $\mathrm{C}-\mathrm{C}$ units is examined from ab initio with an assessment of the properties of chemical bonding. The energy-volume equation of state shows large anisotropy effects due to C-C alignment along tetragonal $c$-axis leading to high linear incompressibility. Relevant features of selective bonding of uranium and cobalt with carbon at two different Wyckoff sites and strong C-C interactions are remarkable. The vibrational frequencies for C...C stretching modes indicate closer behavior to aliphatic $\mathrm{C}-\mathrm{C}$ rather than $\mathrm{C}=\mathrm{C}$ double bond. A ferromagnetic ground state is proposed from the calculations.
\end{abstract}

\section{Keywords}

Uranium cobalt di-carbide. Electronic Structure. EOS. Bonding.

* Correspondence: matar@icmcb-bordeaux.cnrs.fr 


\section{Introduction}

Uranium forms a series of binary compounds with carbon and silicon. The equiatomic UC with rock-salt structure contains isolated $\mathrm{C}$ atoms and no $\mathrm{C}-\mathrm{C}$ bonds, while $\mathrm{U}_{2} \mathrm{C}_{3}$ and $\mathrm{UC}_{2}$ have $\mathrm{C}_{2}$ pairs as carbon substructure. Similarly the higher congener silicon $\mathrm{U}_{3} \mathrm{Si}$ has isolated silicon atoms while $\mathrm{U}_{3} \mathrm{Si}_{2}$ has $\mathrm{Si}_{2}$ pairs [1]. A rare example of mixed uranium silicide carbide is $\mathrm{U}_{3} \mathrm{Si}_{2} \mathrm{C}_{2}[2,3]$ which contains $\mathrm{SiC}$ units. Based on computations within the density functional theory (DFT) [4] the chemical bonding was identified to be specific to uranium sites with $\mathrm{Si}$ and $\mathrm{C}$ with major $\mathrm{Si}-\mathrm{C}$ bonding [5]. At another level of theory Li and Hoffmann [6] discussed the chemical role of C...C units in several binary and ternary metal carbides with extended Hückel methodology, including title compound. X-ray diffraction studies of $\mathrm{UCoC}_{2}$ by Gerss, W. Jeitschko led to assign a new structural type derived from PbFCl structure [7]. In continuation of our ab initio investigations of the electronic and magnetic structures and chemical bonding of uranium based compounds within DFT [5, 8-11], we address herein these properties for $\mathrm{UCoC}_{2}$ with stressing the effect of the structural anisotropy brought by C...C pairs on site selective chemical bonding with metal atoms $\mathrm{U}$ and $\mathrm{Co}$ and on the mechanical properties. Further from energy criteria a ferromagnetic ground state is proposed.

\section{Computational methodologies}

Two computational methods within the DFT were used in a complementary manner. The Vienna ab initio simulation package (VASP) code [12,13] allows geometry optimization and total energy calculations. For this we use the projector augmented wave (PAW) method [13,14], built within the generalized gradient approximation (GGA) scheme following Perdew, Burke and Ernzerhof (PBE) [15]. Also preliminary calculations with local density approximation LDA [16] led to an underestimated volume versus the experiment, i.e. $84 \AA^{3}$ versus $89.4 \AA^{3}$ [7]. The conjugate-gradient algorithm [17] is used in this computational scheme to relax the atoms. The tetrahedron method with Blöchl corrections [14] as well as a Methfessel-Paxton [18] scheme were applied for both geometry relaxation and total energy calculations. Brillouin-zone (BZ) integrals were approximated using the special k-point sampling. The optimization of the structural parameters was performed until the forces on the atoms were less than 0.02 
$\mathrm{eV} / \AA$ and all stress components less than $0.003 \mathrm{eV} / \AA^{3}$. The calculations are converged at an energy cut-off of $400 \mathrm{eV}$ for the plane-wave basis set with respect to the k-point integration with a starting mesh of $4 \times 4 \times 4$ up to $8 \times 8 \times 8$ for best convergence and relaxation to zero strains. The calculations are scalar relativistic.

In the context of the electronic structure investigations of actinides within the DFT, it may be argued that the correlations accounted for within its basic functionals (LDA and GGA) may not be sufficient enough to account for stronger correlations characterizing the $5 f$ states. Then $\mathrm{LDA}+U$ or GGA $+U$ method can be used to reproduce experimental findings. $U$ stands for the Coulomb on-site repulsive parameter added to enhance correlation. Beside plain GGA we used such test calculations following Dudarev et al. [19], with $U=4 \mathrm{eV}$ value which was shown to better approach experiment in $\mathrm{U}_{3} \mathrm{Si}_{2} \mathrm{C}_{2}[6]$.

Then all-electron calculations with the GGA were carried out for a full description of the electronic structure and the properties of chemical bonding, using full potential scalar-relativistic augmented spherical wave (ASW) method [20,21]. In the minimal ASW basis set, we chose the outermost shells to represent the valence states and the matrix elements were constructed using partial waves up to $l_{\max }+1=4$ for $U, l_{\max }+1=3$ for $\mathrm{Co}$ and $\mathrm{l}_{\max }+1=2$ for $\mathrm{C}$. Self-consistency was achieved when charge transfers and energy changes between two successive cycles were below $10^{-8}$ and $10^{-6} \mathrm{eV}$, respectively. BZ integrations were performed using the linear tetrahedron method within the irreducible wedge. In order to optimize the basis set, additional augmented spherical waves are placed at carefully selected interstitial sites (IS). Besides the site projected density of states, we discuss qualitatively the pair interactions based on the overlap population analysis with the crystal orbital overlap population (COOP) [22]. In the plots, positive, negative, and zero COOP indicate bonding, anti-bonding, and nonbonding interactions, respectively.

3. Crystal chemistry The crystal structure of $\mathrm{UCoC}_{2}$, space group $P 4 / \mathrm{nmm}$, is sketched in Fig. 1 and described in Table 1. The unit cell can be considered as a stacking of Co, $\mathrm{U}$ and $\mathrm{C}$...C along the tetragonal $c$-axis. It can be considered as an anti-PbFCl-type because Co occupies the F position at the origin (2a) whereas $\mathrm{U}$ is at $\mathrm{Pb}(2 c)$ with $01 \frac{1}{2} \mathrm{z}$. 
Carbon at chlorine sites (2c) is found within two sites, C1 and C2. It can be noted that this structure is different from actinide based homologue $\mathrm{ThCoC}_{2}$ which crystallizes in the base centered monoclinic $\mathrm{CeNiC}_{2}$-type, indentified for a large number of compounds [23].

The C...C pairs (C1-C2, Fig. 1) have short distance of $\sim 1.47 \AA$ which is between CC single bond of $\sim 1.5 \AA$ and $\mathrm{C}=\mathrm{C}$ double bond (1.34 $\AA$ ). Also the other short distances shown in Table 1 are those of $\mathrm{U}$ with $\mathrm{C} 2$ and Co with $\mathrm{C} 1$. Then significant bonding between them on one hand and between $\mathrm{C} 1$ and $\mathrm{C} 2$ on the other hand can be expected as it is discussed in section 5.2.

\section{Results and discussions}

\subsection{Geometry optimization and equation of state (EOS)}

Table 1 provides the experimental and calculated crystal structure parameters. With GGA the volume is found slightly below experimental value, in better agreement than with preliminary PAW-LDA calculations which severely underestimated it. The $z$ parameters of $\mathrm{U}, \mathrm{C} 1$ and $\mathrm{C} 2$ atoms at $(2 c)$ position are in good agreement with experiment as well as the interatomic distances which are found only slightly smaller. The $3^{\text {rd }}$ column showing the GGA $+U$ calculations lead to better agreement with experiment for the volume and for $z(\mathrm{U})$ but to significant deviations of the $z(\mathrm{C} 1)$ and $z(\mathrm{C} 2)$ parameters as well. The energy is highly raised due to the $U$ repulsive character. Then it may be suggested that the changes are not significant of improvements on all structure properties as it was the case of $\mathrm{U}_{3} \mathrm{Si}_{2} \mathrm{C}_{2}$ [5] and the following results are from calculations using plain PAW-GGA method.

For a better assessment of the results we derive the equilibrium zero pressure parameters from the energy-volume $(E, V)$ equation of state $(E O S)$ with $(E, V)$ set of calculations around minima found from geometry optimization. The underlying physics of this procedure is that the calculated total energy corresponds to the cohesion within the crystal in as far as the solution of the Kohn-Sham DFT equations gives the energy with respect to infinitely separated electrons and nuclei. But the zero of energy depends on the choice of the potentials, then energy becomes arbitrary through its shifting, not scaling. However the energy derivatives as well as the EOS remain unaltered. For this 
reason one needs to establish EOS from which the fit parameters are extracted for an assessment of the equilibrium values. The resulting values are plotted in Fig. 2. They have a quadratic variation which can be fitted with an energy-volume Birch EOS up to the $3^{\text {rd }}$ order [24]:

$$
\mathrm{E}(\mathrm{V})=\mathrm{E}_{0}\left(\mathrm{~V}_{\mathrm{o}}\right)+[9 / 8] \mathrm{V}_{0} \mathrm{~B}_{\mathrm{o}}\left[\left(\left[\left(\mathrm{V}_{\mathrm{o}}\right) / \mathrm{V}\right]\right)^{[2 / 3]}-1\right]^{2}+[9 / 16] \mathrm{B}_{0}\left(\mathrm{~B}^{\prime}-4\right) \mathrm{V}_{\mathrm{o}}\left[\left(\left[\left(\mathrm{V}_{0}\right) / \mathrm{V}\right]\right)^{[2 / 3]}-1\right]^{3},
$$

where $E_{0}, V_{0}, B_{0}$ and $B$ are the equilibrium energy, the volume, the bulk modulus and its pressure derivative, respectively. The fit curves reproduce the trends of the geometry optimization for the energies and the volumes and provide the results given in the insert. It can be noted that the volume is now closer to experiment. The corresponding zero pressure bulk modulus of $\mathrm{B}_{0}=260 \mathrm{GPa}$ positions the hardness of the compound within the range of oxides. However in view of the anisotropic structure (Fig.1) with C-C alignments we further investigated the linear compressibility along the $c$-axis. The obtained E,V curve shows a steeper change of the energy with volume leading to different fit parameters with the Birch EOS given above: the bulk modulus is strongly enhanced, $\mathrm{B}_{0}=560 \mathrm{GPa}$, with respect to isotropic volume compression while the equilibrium volume $\mathrm{V}_{0}$ and energy $\mathrm{E}_{0}$ show little change. This result may be assigned to the incompressibility of aligned C-C substructures with U. B' values are within range of usually encountered magnitudes [25].

\subsection{Elastic stiffness constants}

In tetragonal symmetry there are six independent elastic stiffness constants $C_{11}, C_{33}$, $\mathrm{C}_{44}, \mathrm{C}_{66}, \mathrm{C}_{12}$ and $\mathrm{C}_{13}$. Most encountered compounds are polycrystalline where monocrystalline grains are randomly oriented so that on a large scale, such materials can be considered as statistically isotropic. They are then completely described by the bulk modulus B and the shear modulus G, which may be obtained by averaging over the single-crystal elastic constants. The most widely used averaging method is Voigt's based on a uniform strain (cf. [26] for a review with therein refs.). The calculated elastic constants in $\mathrm{UCoC}_{2}$ are (in units of GPa):

$\mathrm{C}_{11}=\mathrm{C}_{22}=358 ; \mathrm{C}_{12}=241 ; \mathrm{C}_{13}=149 ; \mathrm{C}_{33}=530 ; \mathrm{C}_{44}=206$ and $\mathrm{C}_{66}=144$.

All $\mathrm{C}_{\mathrm{ij}}$ are positive and their combinations: $\mathrm{C}_{11}>\mathrm{C}_{12}, \mathrm{C}_{11} \mathrm{C}_{33}>\mathrm{C}_{13}{ }^{2}$ 
and $\left(\mathrm{C}_{11}+\mathrm{C}_{12}\right) \mathrm{C}_{33}>2 \mathrm{C}_{13}{ }^{2}$, obey the rules pertaining to the mechanical stability of the chemical system.

$\mathrm{C}_{33}$ is much larger than $\mathrm{C}_{11}=\mathrm{C}_{22}$, in agreement with the high linear incompressibility along the $c$ axis, i.e. in the direction of C...C pairs.

The bulk $\left(\mathrm{B}_{\mathrm{V}}\right)$ and shear $\left(\mathrm{G}_{\mathrm{V}}\right)$ modules following Voigt are formulated as:

$\mathrm{B}_{\mathrm{V}}=1 / 9\left\{2\left(\mathrm{C}_{11}+\mathrm{C}_{12}\right)+4 \mathrm{C}_{13}+\mathrm{C}_{33}\right\}$

$\mathrm{G}_{\mathrm{V}}=1 / 30\left\{12 \mathrm{C}_{44}+12 \mathrm{C}_{66}+\mathrm{C}_{11}+\mathrm{C}_{12}+2 \mathrm{C}_{33}-4 \mathrm{C}_{13}\right\}$

The resulting numerical values are: $\mathrm{B}_{\mathrm{V}}=258 \mathrm{GPa}$ and $\mathrm{Gv}=176 \mathrm{GPa}$. It is interesting to see that the bulk modulus obtained with the formula based on the elastic constants comes close to the one obtained by Birch EOS. This validates a posteriori the two approaches in analyzing the mechanical properties of the compound.

The shear modulus which defines the rigidity of the material is significantly lower than the bulk modulus and the ratio G/B which is an indicator of brittleness/ductility has a value of 0.7. This defines a brittle material as for instance Ir metal contrary to neighboring transition metals $\mathrm{Ag}, \mathrm{Pt}$, au with $\mathrm{G} / \mathrm{B}$ ratios in the range $\{0.4-0.2\}$ [27].

\subsection{Analysis of the charge trends}

Analyzing the charge density issued from the self consistent calculations can be done using the AIM (atoms in molecules theory) approach [28] developed by Bader who devised an intuitive way of splitting molecules into atoms as based purely on the electronic charge density. Typically in chemical systems, the charge density reaches a minimum between atoms and this is a natural region to separate them from each other. Such an analysis does not constitute a tool for evaluating absolute ionizations. The results of computed charge changes $(\Delta \mathrm{Q})$ are such that they lead to neutrality when the respective multiplicities are accounted for:

$$
\mathrm{U}:+1.78 \text {; Co: +0.40; C1: }-0.95 \text { and C2: }-1.22 \text {. }
$$

These original results show different behaviors of carbon at the C1 and C2 sites with resulting charge difference of $\delta=|0.27|$. A C...C bond dipole moment (in Debye unit) is obtained: $\mu=\mathrm{d} \delta=1.47 \times 0.27=0.4 \mathrm{D}$. Despite its small magnitude compared with 
ionic compounds such as $\mathrm{KBr}\left(\mathrm{K}^{+} \mathrm{Br}^{-}\right.$with $\left.\mu=10 \mathrm{D}\right)$, this stresses the role of differentiated interactions and selective charge transfers of $\mathrm{C} 1$ and $\mathrm{C} 2$ with Co and $\mathrm{U}$ respectively through the chemical bond.

We further assess the nature of the C...C bond with the calculation of the vibrational frequencies of the whole crystal system calculated through the Hessian matrix using finite differences with VASP package with parameter IBRION $=6$ in INCAR control file (cf. web ref. in [13]). The results are such that at low frequencies $\left(\sim 150 \mathrm{~cm}^{-1}\right)$ the lattice modes are found involving the displacements of the whole structure in a rigid manner and at the highest frequencies the C...C antisymmetric and symmetric stretching modes are obtained with $v(C \ldots C)=958 \mathrm{~cm}^{-1}$ and symmetric $v(C \ldots C)=1000$ $\mathrm{cm}^{-1}$ respectively. Screen snapshots from the relevant stretching animations are shown in Fig. 3 with arrows indicating the directions. The highest value comes closer to infra red (IR) frequency for aliphatic C-C with $v(I R)=1200 \mathrm{~cm}^{-1}$ than ethylene like $\mathrm{C}=\mathrm{C}$ with $v(I R)=1650 \mathrm{~cm}^{-1}$. Note that a better agreement cannot be expected in as far as the vibration frequencies were calculated in the solid state whereas the text books refer to $\mathrm{C}-\mathrm{C}$ and $\mathrm{C}=\mathrm{C}$ in molecules.

\section{Electronic structure and chemical bonding}

\subsection{Electronic density of states.}

Calculations used the experimental parameters with the full potential scalar relativistic ASW method in which chemical bonding qualitative descriptions implementations were done by V. Eyert following different schemes [21].

Firstly spin degenerate (non spin polarized NSP) calculations with total spins are done. At self consistent convergence the charge transfer follows the trends observed above with additional charge residues (corresponding to less than 0.15 electrons) from the atomic spheres to IS. The site projected densities of states (PDOS) are shown in Fig. 4a. In both panels, the zero energy along the $x$-axis is considered with respect to the Fermi level $E_{F}$ in as far as the ternary compound is metallic due to the PDOS contributions from all constituents, especially the lower part of the uranium $5 f$ states. Due to their low filling $\mathrm{U}(5 f)$ are centered above $\mathrm{E}_{\mathrm{F}}$. The opposite is observed for 
largely filled d states of Co which are centered below $E_{\mathrm{F}}$. Low energy lying carbon $s$ at $\sim 15 \mathrm{eV}$ are followed by $p$ PDOS around $-10,-5$ and from -4 up to $\mathrm{E}_{\mathrm{F}}$ with similar shapes to $\mathrm{U}$ and Co PDOS, thus ensuring for the chemical bonding with them.

While cobalt shows a small magnitude PDOS at $\mathrm{E}_{\mathrm{F}}$, the lower part of $\mathrm{U}(5 f)$ crosses $\mathrm{E}_{\mathrm{F}}$ at a large PDOS. This signals instability of the compound for uranium $f$ states in such spin degenerate magnetic configuration within the Stoner mean field theory of band ferromagnetism [29] which can be applied to address the tendency for spin polarization (not the long range magnetic order). The total energy of the spin system results from the exchange and kinetic energies. Referring the total energy to the non-magnetic state (NSP), this is expressed as: $\mathrm{E}=$ constant $\left\{1-\mathrm{I} n\left(\mathrm{E}_{\mathrm{F}}\right)\right\}$. In this expression, $\mathrm{I}(\mathrm{eV})$ is the Stoner integral and $n\left(\mathrm{E}_{\mathrm{F}}\right)(1 / \mathrm{eV})$ is the PDOS value for a given state -here $f$ - at the Fermi level in the non-magnetic state (Fig. 4a). If the unit-less Stoner product I.n( $\mathrm{E}_{\mathrm{F}}$ ) is larger than 1, energy is lowered and the system stabilizes in a magnetically ordered configuration. Then the product I. $n\left(\mathrm{E}_{\mathrm{F}}\right)$ provides a criterion for the stability of the spin system. From quantum theoretical calculations [30] the value of $\mathrm{I}\{\mathrm{U}(5 f)\}=0.51 \mathrm{eV}$ was derived. With $n\left(\mathrm{E}_{\mathrm{F}}\right)$ value of $3.8 \mathrm{eV}^{-1}$ the calculated $\operatorname{I} n\left(\mathrm{E}_{\mathrm{F}}\right)$ is 1.94 and the Stoner criterion 1-I $n\left(\mathrm{E}_{\mathrm{F}}\right)$ of -0.94 is negative, leading to energy lowering upon the onset of magnetization. Spin polarized (SP) calculations actually lead to a lowering of the total energy by $2.1 \mathrm{eV}$ and to a total magnetization $1.3 \mu_{\mathrm{B}}$ /formula unit in a ferromagnetic configuration. The major contribution to total magnetization arises from uranium with $\mathrm{M}(\mathrm{U})=1.24 \mu_{\mathrm{B}}$ while $\mathrm{M}(\mathrm{Co})=0.06 \mu_{\mathrm{B}}$. Such magnetic polarization was suggested [5, 7] without further specification. The effect on the PDOS is shown at Fig. 4b. In spin polarized calculations with DFT-GGA the magnetic moment arises from the rigid band shift between majority $\Uparrow$ spin populations and minority $\Downarrow$ spins ones through magnetic exchange. This is observed mainly for uranium $f$-PDOS around $\mathrm{E}_{\mathrm{F}}$ while the other constituents PDOS exhibit small (Co) or no (C1, C2) energy shift.

However the spin only value obtained from scalar relativistic calculations should be corrected by the spin orbit coupling SOC with the orbital moment which amounts to 2.7 in uranium [11]. This obeys Hund's $3^{\text {rd }}$ rule whereby the actual quantum number is $\mathrm{J}$ with $\mathrm{J}=|\mathrm{L}-\mathrm{S}|$ for less than half filled $f$ subshell and $\mathrm{J}=|\mathrm{L}+\mathrm{S}|$ for more than half filled 
subshell. The first condition corresponds to uranium and the resulting moment is $2.4 \mu_{\mathrm{B}}$. This should be confronted with experiment when available.

For the search of the actual magnetic ground state, an antiferromagnetic configuration was tested with dispatching the crystal into two magnetic subcells, one of them for $\Uparrow$ SPIN and the other for $\Downarrow$ SPIN. The result is zero magnetization as expected, and a raise of the energy by $+0.5 \mathrm{eV} / \mathrm{UCoC}_{2}$ unit. Thus the magnetic ground state is predicted to be ferromagnetic. Lastly we note that the magnitude of magnetization only slightly changed with GGA+U calculations $(U=4 \mathrm{eV})$ as in $\mathrm{U}_{3} \mathrm{Si}_{2} \mathrm{C}_{2}$ [6]. This suggests band magnetism behavior of $\mathrm{UCoC}_{2}$ ternary, due to the presence a transition metal of the first period $\mathrm{T}$, (here Co) besides uranium like in $\mathrm{UT}_{2} \mathrm{X}_{2}(\mathrm{X}=p$ element) $[9,10]$.

\subsection{Chemical bonding.}

Fig. 5 shows the chemical bonding for pair interactions with the COOP criterion [19] as implemented within the ASW method [18]. They are based on non magnetic calculations in as far as the spin polarized calculation have the role of rigidly shifting the majority spins PDOS to lower energy and the minority spin PDOS to higher energy (Fig. 4b). The major part of the valence band (below $E_{F}$ ) is of bonding character with small antibonding COOP (negative magnitudes) from $-4 \mathrm{eV}$ up to $\mathrm{E}_{\mathrm{F}}$ arising from C1$\mathrm{C} 2$ bonds due to their involving with $\mathrm{U}$ and Co bonding. The energy window is reduced with respect to the DOS panel in order to better show the pair interactions and because no significant bonding could be identified below $-11 \mathrm{eV}$.

The major pair-bonding is in the energy range $-8,-5 \mathrm{eV}$ i.e. where $\mathrm{C}-p$ states are dominant. As expected the carbon pair (C1-C2) bonding prevails, followed by Co-C1 and U-C2 while the other interactions are of weaker intensity. U-Co bonding is also present especially in the energy range $-4,-1 \mathrm{eV}$ where Co-d states mix with uranium itinerant states below $\mathrm{E}_{\mathrm{F}}$ but it is weaker than the other intensities due to the large U-Co distance: $\sim 3.6 \AA$. These bonding intensity follow from the crystal environments and the subsequent distances (Table 1). It is likely that the brittleness of the compound arises from such strong directional and site specific interactions. 


\section{Conclusions}

In this work further evidence of the effects of C...C pairs in the building of the ternary carbide $\mathrm{UCoC}_{2}$ was provided. The structural anisotropy arising from their alignment along the tetragonal $c$-axis leads to assign a high linear incompressibility as inferred from the energy-volume curves fitted with Birch EOS as well as from the set of elastic constants leading to assign a brittle like behavior. The chemical bonding is specific to carbon sites (C1 and C2) with cobalt and uranium respectively, and large C1$\mathrm{C} 2$ and weak U-Co bonding. The nature of the C-C bond is found from the calculations of the frequencies to be closer to aliphatic C-C single bond. Further it is found that a dipole moment is developed due to the differentiated bonding of C1 and C2 respectively with Co and U. From energy differences the ground state is predicted ferromagnetic with major contribution to the magnetization arising from uranium spin polarization. Future works will be devoted to experimental characterizations of the magnetic properties aided by full relativistic calculations.

Acknowledgements: Parts of the calculations were carried out on AVAKAS cluster of MCIA-Mesocenter of the University Bordeaux 1.

\section{References}

[1] P. Rogl, H. Noël, J. Phase Equilibria 16 (1995) 66.

[2] P. L. Blum, P. Guinet, G. Silvestre, Compt. Rend. Acad. Sci. Paris, Ser. C 260 (1965) 1911.

[3] R. Pöttgen, D. Kaczorowski, W. Jeitschko, J. Mater. Chem. 3 (1993) 253.

[4] P. Hohenberg, W. Kohn, Phys. Rev. 136 (1964) B864

and W. Kohn, L. J. Sham, Phys. Rev. 140 (1965) A1133.

[5] J. Li, R. Hoffmann, Chem. Mater., 1 (1989) 83.

[6] S.F. Matar, R. Pöttgen, Chem. Phys. Lett., 550 (2012) 88.

[7] M.H. Gerss, W. Jeitschko, Mat. Res. Bull., 21 (1986) 209.

[8] S. F. Matar, A.F Al Alam, New. Journal of Physics (IOP): 10 (2008) 083013 (11pp).

[9] S. F. Matar, V. Siruguri, J. Alloys Comp. 436 (2007) 34.

[10] S. F Matar, V. Siruguri, V. Eyert, J. Magn. Magn. Mater. 305 (2006) 264. 
[11] S. F. Matar, A. Mavromaras, J. Solid State Chem. 149 (2000) 449.

[12] G. Kresse, J. Furthmüller, Phys. Rev. B 54 (1996) 11169.

[13] G. Kresse, J. Joubert, Phys. Rev. B 59 (1999) 1758.

http://cms.mpi.univie.ac.at/vasp/vasp/IBRION_5_IBRION_6.html

[14] P. E. Blöchl, Phys. Rev. B 50 (1994) 17953.

[15] J. Perdew, K. Burke, M. Ernzerhof, Phys. Rev. Lett. 77 (1996) 3865.

[16] D. M. Ceperley, B. J. Alder, Phys. Rev. Lett. 45 (1980) 566.

[17] W.H. Press, B.P. Flannery, S.A. Teukolsky, W.T. Vetterling, Numerical Recipes, Cambridge University Press, New York (1986).

[18] M. Methfessel, A. T. Paxton, Phys. Rev. B 40 (1989) 3616.

[19] S. L. Dudarev, G. A. Botton, S. Y. Savrasov, C. J. Humphreys, A. P. Sutton, Phys. Rev. B 57 (1998) 1505.

[20] A. R. Williams, J. Kübler, C. D. Gelatt, Phys. Rev. B 19 (1979) 6094.

[21] V. Eyert, The Augmented Spherical Wave Method - A Comprehensive Treatment, Lecture Notes in Physics, Springer, Heidelberg (2007).

[22] R. Hoffmann, Angew. Chem. Int. Ed. Engl. 26 (1987) 846.

[23] W. Jeitschko, M.H. Gerss, J. Less Common Metals, 116 (1986) 147.

[24] F. Birch, J. Geophys. Res. 83 (1978) 1257.

[25] S. F. Matar, G. Demazeau, M. H. Möller, R. Pöttgen, Chem. Phys. Lett. 508 (2011) 21.

[26] S. F. Matar, Matériaux ultra-durs: Concepts et Modélisations. Série Techniques de l'ingénieur. Eds. T. I. Sciences et Techniques. Paris. - AF 6630 (20pp) 2009.

[27] S. Kamran, K. Chen, L. Chen, L. Zhao, J. Phys. Condens. Matter. 20 (2008) 085221

[28] R. Bader, Chem. Rev. 91 (1991) 893.

[29] P. Mohn, Magnetism in the solid state - An introduction, Springer Series, in: Solid-State Sciences. M. Cardona, P. Fulde, K. von Klitzing, R. Merlin, H.J. Queisser, H. Störmer (Eds.). Springer, Heidelberg (2003).

[30] S F. Matar, V. Eyert, B. Chevalier, J. Etourneau Int J Quantum Chem., 61 (1997) 705. 
Table 1

Experimental and calculated structural parameters of $\mathrm{UCoC}_{2}$

\begin{tabular}{|c|c|c|c|}
\hline $\begin{array}{l}\mathrm{UCoC}_{2} \\
P 4 / n m m \text { (Orig. 1) }\end{array}$ & Exp. [7] & Calc. PAW-GGA & $\mathrm{GGA}+U(U=4 \mathrm{eV})$ \\
\hline$a(\AA)$ & 3.497 & 3.473 & 3.481 \\
\hline$c(\AA)$ & 7.313 & 7.275 & 7.372 \\
\hline $\mathrm{V}\left(\AA^{3}\right)$ & 89.43 & 87.75 & 89.34 \\
\hline $\begin{array}{l}\text { Co (2a) } 000 \\
\text { U,C1,C2 (2c) } 0 \text { 1/2 z } \\
\text { z(U) } \\
\text { z(C1) } \\
\text { z(C2) }\end{array}$ & $\begin{array}{l}0.321 \\
0.8416 \\
0.6396\end{array}$ & $\begin{array}{l}0.319 \\
0.843 \\
0.640\end{array}$ & $\begin{array}{l}0.321 \\
0.849 \\
0.649\end{array}$ \\
\hline $\begin{array}{l}\text { Short distances }(\AA) \\
\text { U-C2 } \\
\text { Co-C1 } \\
\text { C1-C2 }\end{array}$ & $\begin{array}{l}2.33 \\
2.09 \\
1.48 \\
\end{array}$ & $\begin{array}{l}2.34 \\
2.08 \\
1.47 \\
\end{array}$ & $\begin{array}{l}2.41 \\
2.07 \\
1.48 \\
\end{array}$ \\
\hline $\mathrm{E}(\mathrm{eV}) / 2 \mathrm{FU}$ & - & -74.97 & -67.47 \\
\hline
\end{tabular}

FU: formula unit. 


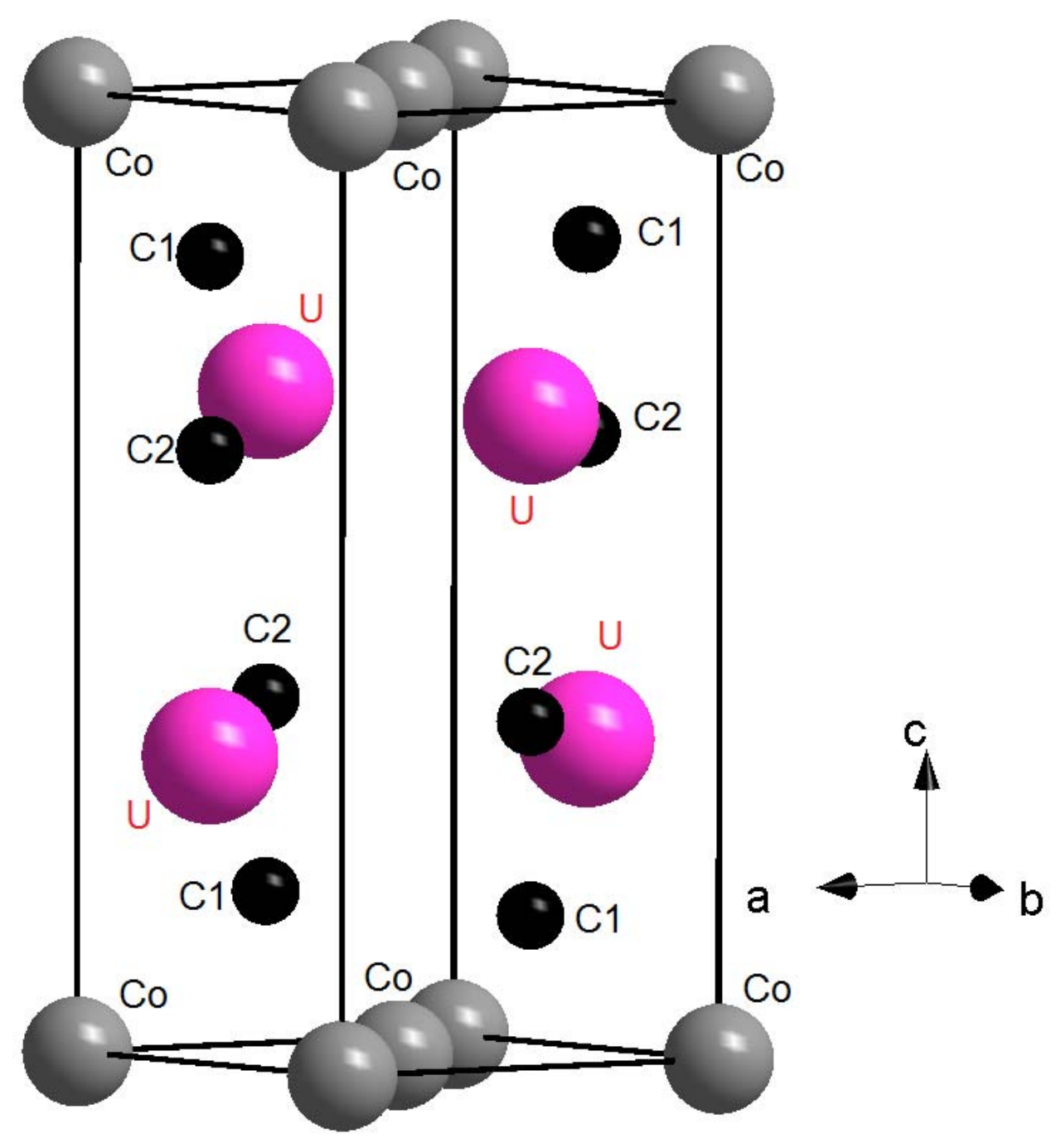

Fig. 1. (color) $\mathrm{UCoC}_{2}$ : Sketch of the crystal structure including two formula units showing the C...C (C1-C2) pairs aligned along the tetragonal $c$-axis. Atom labels are as in Table 1. 


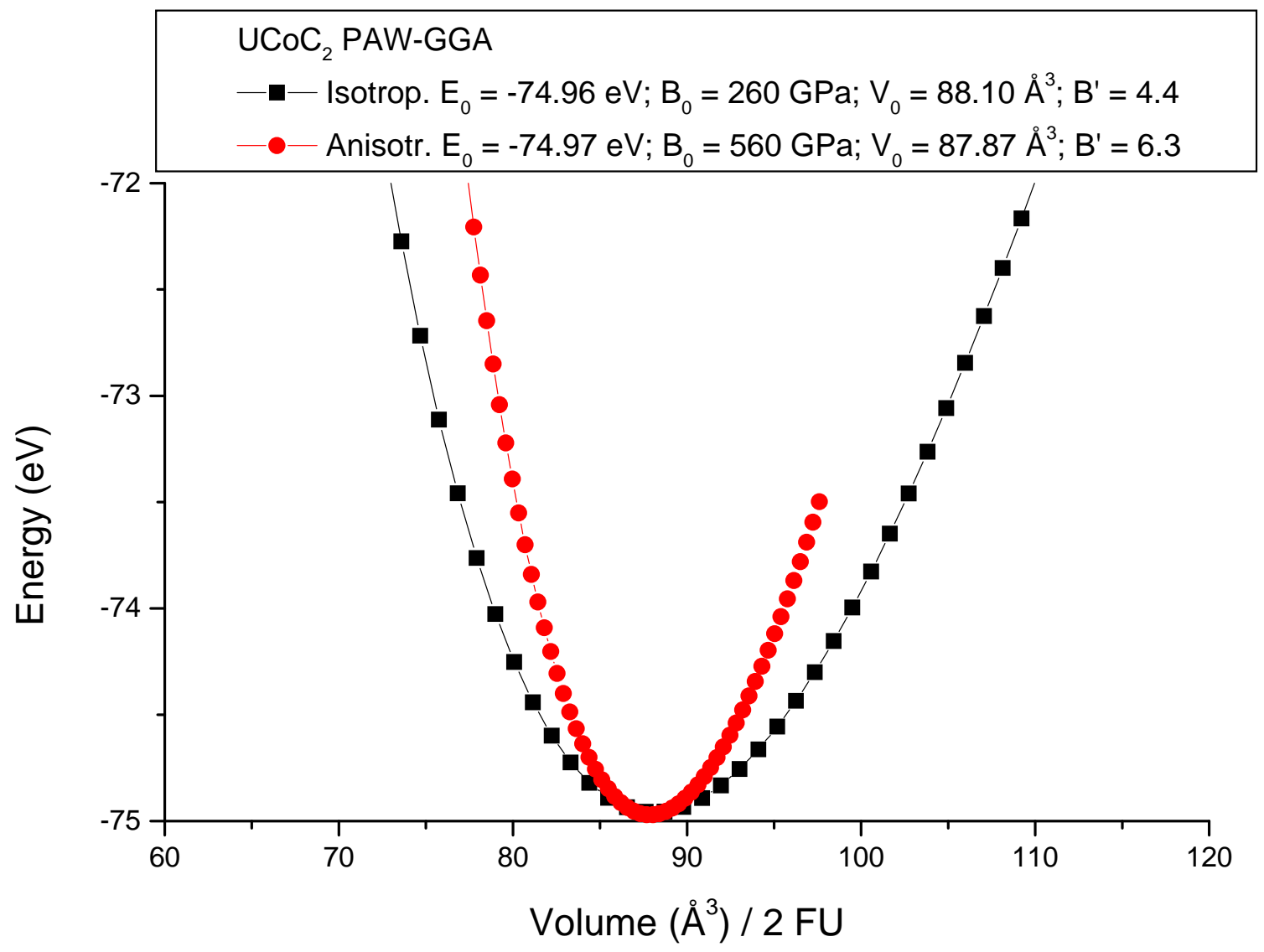

Fig. 2. $\mathrm{UCoC}_{2}$ : Energy volume curves for isotropic and anisotropic volume compressions and corresponding fit parameters (insert) from Birch $3^{\text {rd }}$ order EOS. 

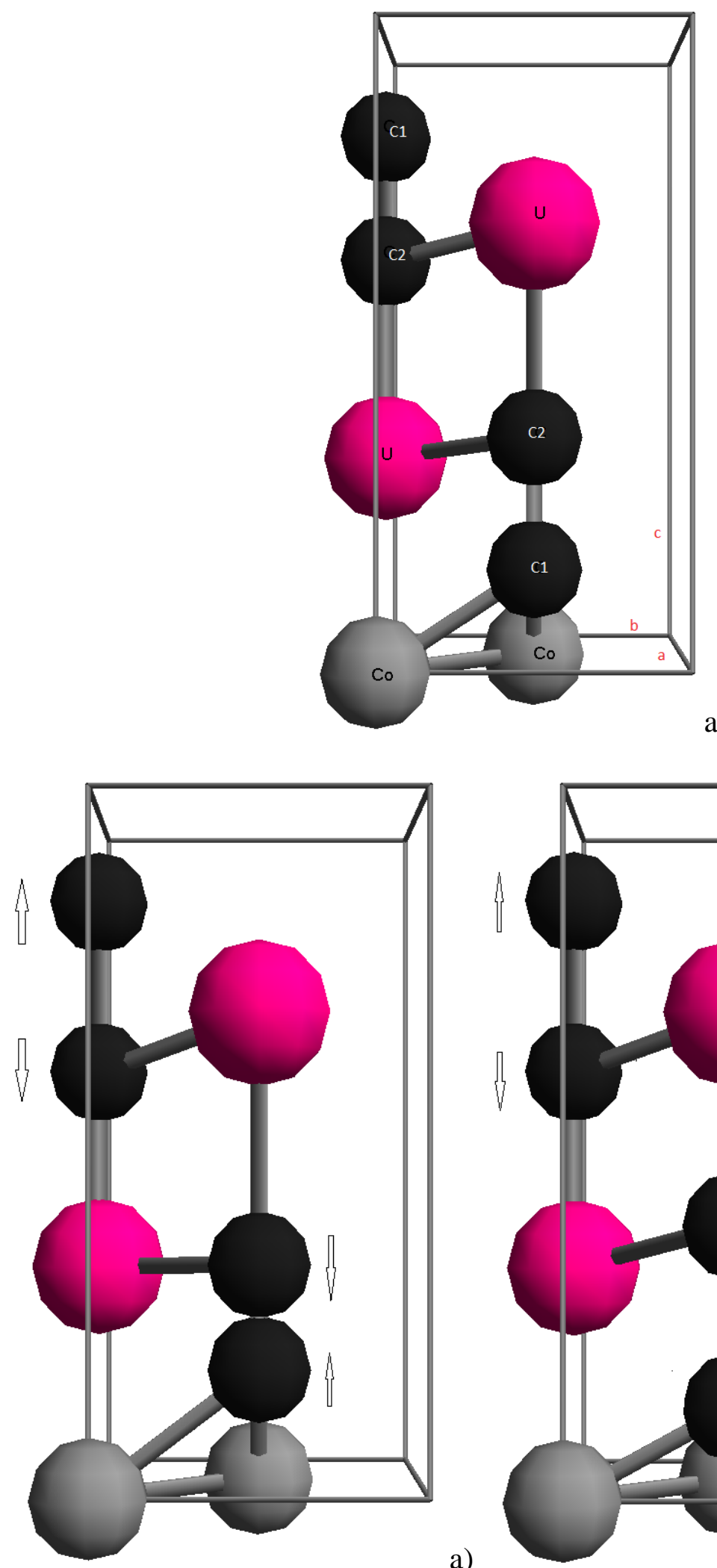

a)

a)

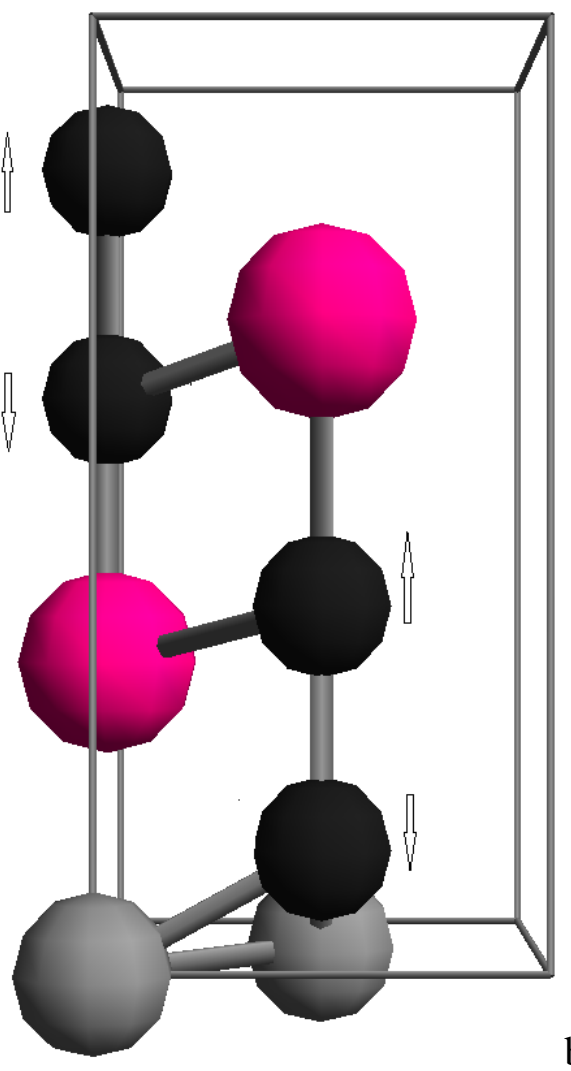

b)

Fig. 3. UCoC2: a) Sketch of the structure with atom labels and snapshots from the animations of b) antisymmetric and c) symmetric C...C stretching modes. 


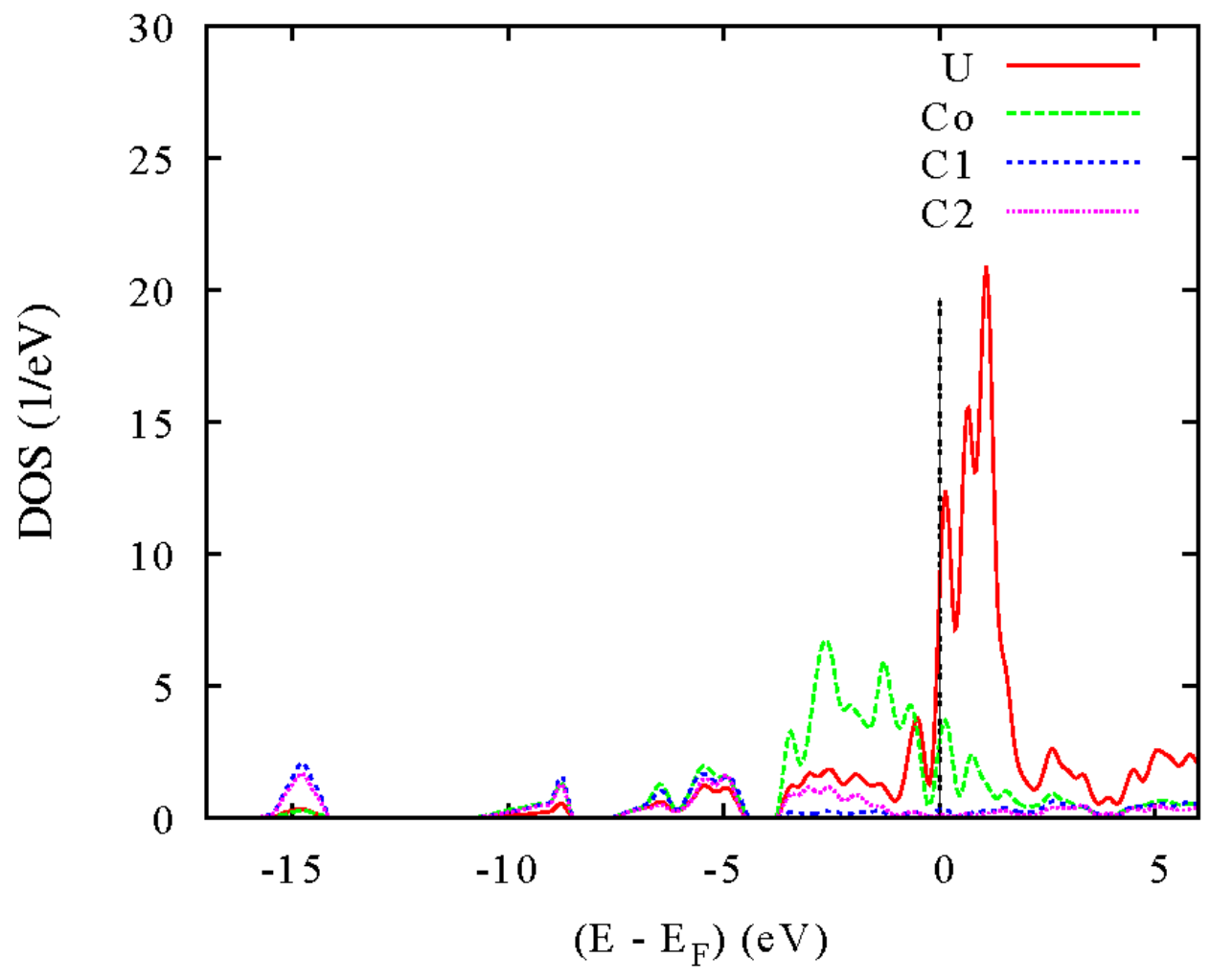

a)

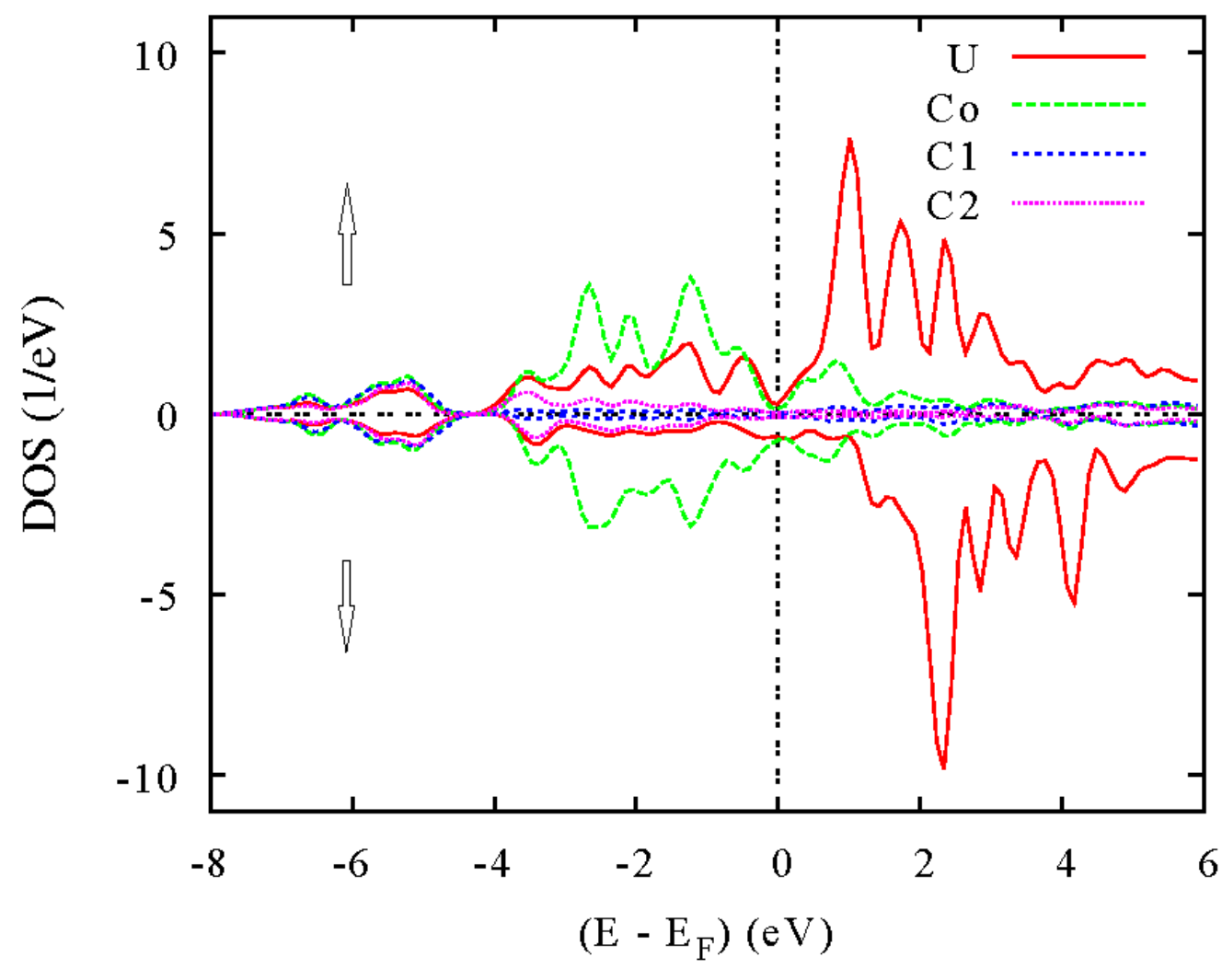

b)

Fig. 4. (color) $\mathrm{UCoC}_{2}$ : a) Spin degenerate non magnetic and b) spin polarized ferromagnetic site projected density of states (PDOS) 


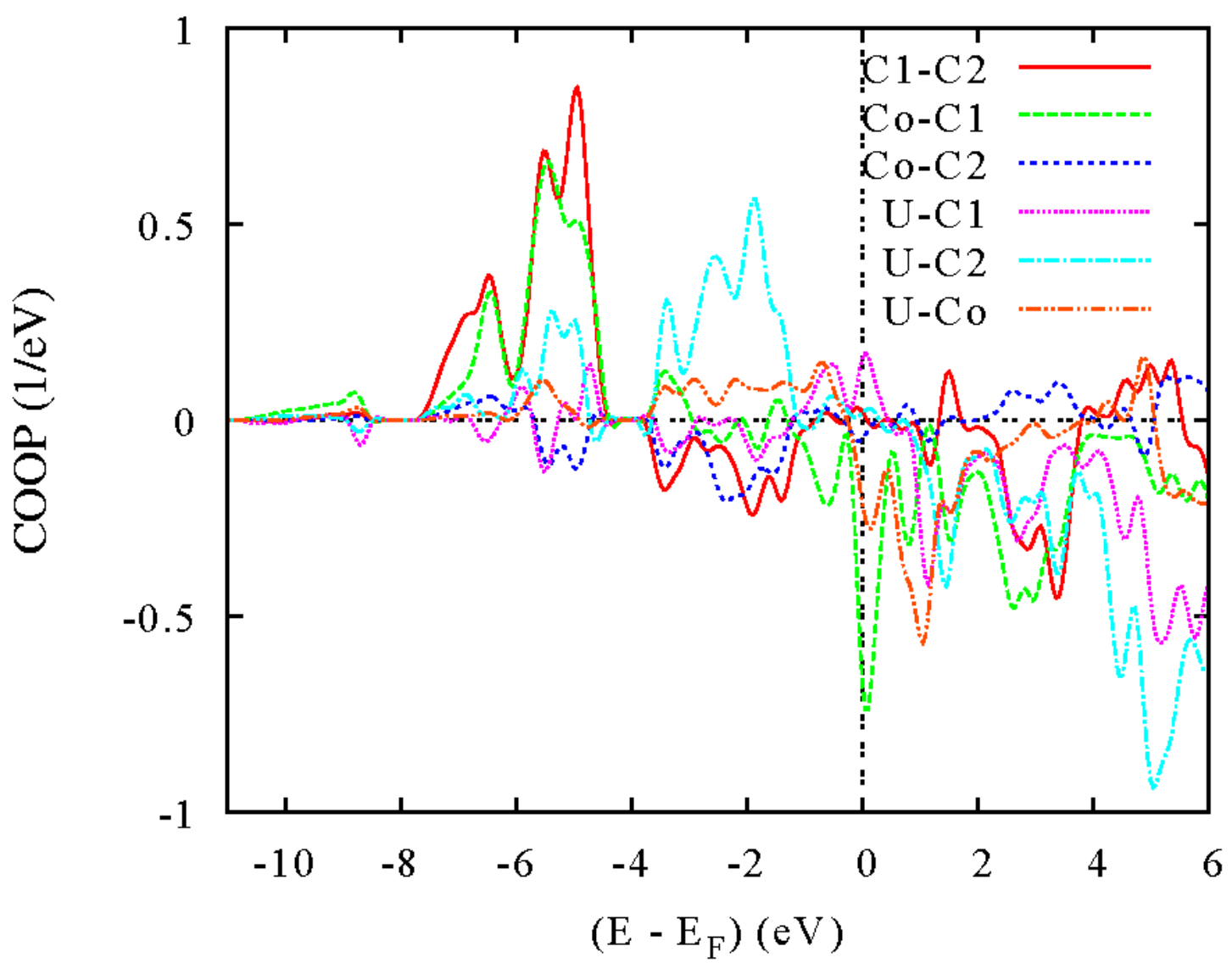

Fig. 5. (color) $\mathrm{UCoC}_{2}$ : chemical bonding for pair interactions using COOP criterion. 